\title{
Perinatal Propylthiouracil-Induced Hypothyroidism Impaired Motor Coordination in Adult Female Offspring
}

\author{
Miski A. Khairinisa ${ }^{1,2}$, Yusuke Takatsuru ${ }^{3}$, Izuki Amano ${ }^{1}$, Michifumi Kokubo ${ }^{1}$, \\ Asahi Haijima ${ }^{4}$, Wataru Miyazaki ${ }^{5}$, Noriyuki Koibuchi ${ }^{1}$ \\ ${ }^{1}$ Department of Integrative Physiology, Gunma University \\ Graduate School of Medicine, Maebashi, Gunma, Japan \\ ${ }^{2}$ Program Study of Pharmacy, Faculty of Mathematics and Natural Sciences \\ Universitas Islam Bandung, Bandung, West Java, Indonesia \\ ${ }^{3}$ Department of Medicine, Johmoh Hospital, Maebashi, Gunma, Japan \\ ${ }^{4}$ Department of Health Sciences and Social Welfare, School of Human Sciences, \\ Waseda University, Saitama, Japan \\ ${ }^{5}$ Department of Bioscience and Laboratory Medicine Hirosaki University, \\ Graduate School of Health Sciences, Hirosaki, Aomori, Japan
}

\begin{abstract}
Thyroid hormone (TH) play crucial roles in the growth and development of brain. Efficiency of TH during the perinatal period results in severe mental and physical retardation, known as cretinism in humans. Animal models have largely focused on mild and severe hypothyroidism associated with deficits in body weight, developmental delays, and cognitive deficits. Although various behavioral analyses have been reported, the effect of perinatal hypothyroidism in adult female mice has not been clarified. The aim of this study was to examine whether propylthiouracil (PTU)-induced hypothyroidism could impair motor coordination in female offspring. We used C57BL/6j mice and divided them into three groups based on the dose of PTU which was applied during perinatal period (embryonic day-14 to postnatal day-14); control, $5 \mathrm{ppm}$, and $50 \mathrm{ppm}$ groups. We observed motor coordination function and additional nociceptive test in female offspring. We found that motor coordination and nociceptive threshold were affected in $50 \mathrm{ppm}$ groups. We concluded that the moderate hypothyroidism could impair motor coordination in adult offspring.
\end{abstract}

Keywords: moderate hypothyroidism, motor coordination

\section{Introduction}

Thyroid hormone insufficiency during fetal and neonatal period impairs brain development. ${ }^{1,2} \mathrm{TH}$ is produced in the thyroid gland, through the iodination of tyrosine residues and coupling of iodotyrosine in the glycoprotein thyroglobulin. Secretion of TH is regulated by thyroid stimulating hormone (TSH) from the anterior pituitary. ${ }^{3}$

Maternal $\mathrm{TH}$ is the only source for fetus during the first trimester of gestation until birth. Severe reduction of TH during gestation is commonly associated with deficiency of iodine, which is an essential compound to synthesize TH. Reduced TH supply to fetus may cause mental retardation, deaf-mutism, motor dysfunction and cognitive impairment,

Corresponding author: Miski A. Khairinisa. Department of Integrative Physiology, Gunma University Graduate School of Medicine, Japan. Email: miskiaghniak@gmail.com

Received: 22 October 2019. Revised: 5 December 2019. Published: 20 December 2019. 
which are sometimes irreversible even with early TH treatment after birth. ${ }^{4,5,7,8}$

Hypothyroid animal models, which are induced by administration of anti-thyroid drug such as PTU, are commonly used to study the role of $\mathrm{TH}$ on brain development. This drug inhibits TH synthesis by inhibiting thyroid peroxidase (TPO) activity. PTU treatment in perinatal period dramatically affects cerebellar development in rodent. ${ }^{6}$ In the present study, we examined whether PTU-induced hypothyroidism could impair motor coordination in female offspring by conducted rotarod test and von Frey hair test as additional examination.

\section{Methods}

Animals

The animal experimentation in this study was approved by the Animal Care and Experimentation Committee of Gunma University. Mice (C57BL/6 strain) used in this study were bred in the Bioresource Center of Gunma University Graduate School of Medicine.

\section{Treatment Schedule}

Animal treatment schedule is shown in Figure 1. Adult male and female mice were mated and the pregnancy was confirmed as embryonic day (ED) 1. Dams were administered 5 or $50 \mathrm{ppm}$ of PTU in drinking water from ED 14 to postnatal day (PND)
21. Control mice were administered distilled water. Dams and all female offspring were separated at weaning day (PND 28). Motor coordination and nociceptive threshold test were conducted at PND 70.

\section{Weight Measurement}

Female offspring were weighed at PND 7, PND 14, PND 21, and PND 28.

\section{Rotarod Test}

Rotarod test was performed to determine motor coordination and motor learning. Mice were placed on the cylinder drum of a rotarod apparatus (LE 8300, LSI Letica Scientific Instruments, Barcelona, Spain). Before the test, mice were habituated for 1 minute. The apparatus was started at a speed of $4 \mathrm{rpm}$ and was accelerated to $40 \mathrm{rpm}$. The mice performed 5 trials per day. To evaluate motor learning, the test was performed for 3 consecutive days.

\section{von Frey hair test}

von Frey hair test was conducted to determine nociceptive threshold. Mechanical force was applied using von Frey hairs with different buckle forces $(0.2-4.0 \mathrm{~g})$. Mice were individually placed on the platform, and filaments were applied to the hind paw. We performed 5 trials with 30 seconds intervals. The mechanical nociceptive threshold was defined as the lowest force that evoked paw withdrawal in at least $50 \%$ of the trials.

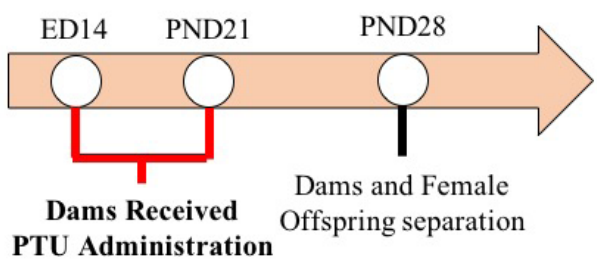

Female Offspring

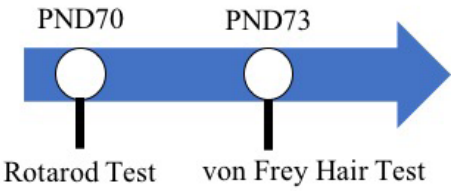

Figure 1. Animal Treatment Schedule 


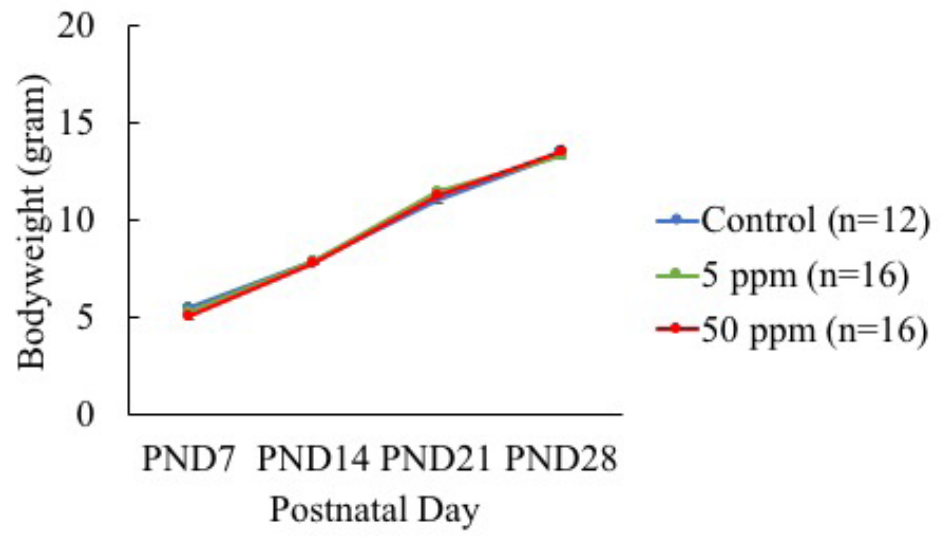

Figure 2. Bodyweight Measurement

\section{Statistical Analysis}

Statistical comparisons were performed by one- way ANOVA with Bonferroni post hoc test using SPSS Software version 22.0 (IBM SPSS, Armonk, NY, USA). Differences were considered significant at $p<0.05$. All values are presented as the mean \pm SEM

\section{Results and Discussion}

This study is part of the author's research project in which previous results have been published. ${ }^{2} \mathrm{TH}$ status has been measured and $\mathrm{TH}$ levels on $5 \mathrm{ppm}$ groups tend to decrease, although not statistically significant. On the other hand, $50 \mathrm{ppm}$ groups showed low levels. Thus, we classified 5 ppm as "mild" and 50ppm as "moderate" hypothyroid mice model.

\section{Bodyweight Measurement}

$\mathrm{TH}$ plays an essential role in bodyweight regulation through regulating energy expenditure $^{9,10}$ Study in human, thyroid dysfunction cause significant changes in body weight and resting metabolic rate. ${ }^{10,11}$ Study in rat showed that PTU at 3 ppm dose altered bodyweight of pups ${ }^{12}$, whereas no significant decrease have found in mice. ${ }^{1}$ In present study, PTU administration in perinatal period did not decrease offspring's bodyweight (Figure 2), which is consistent with the previous study in mice. ${ }^{1}$ However, the mechanism underlying $\mathrm{TH}$ and bodyweight changes are not yet fully understood.

\section{Perinatal Hypothyroidism Impaired Motor Coordination}

As shown in Figure 3, the time spent on the rotarod was significantly decrease in PTUtreated groups in three consecutive days. Several brain regions are involved in motor coordination, but cerebellum plays the major role. TH regulates cerebellum function. Perinatal hypothyroidism reduced the growth and branching of Purkinje cell dendrites and the number of synapses between Purkinje cell dendrite and granule cell axons. ${ }^{13}$ Abnormal cerebellar function may induce aberrant motor coordination.

Perinatal Hypothyroidism Decreased Nociceptive Threshold

Hypothyroidism decreased nociceptive threshold in 50 ppm groups (Figure 4). vonFrey test reflects somatosensory cortex function. Sufficient maternal thyroid hormones may be necessary to determine a normal nociceptive function of offspring. ${ }^{14}$ Previous study showed that hypersensitivity may alter motor coordination, and avoidance behaviour. ${ }^{15}$ 


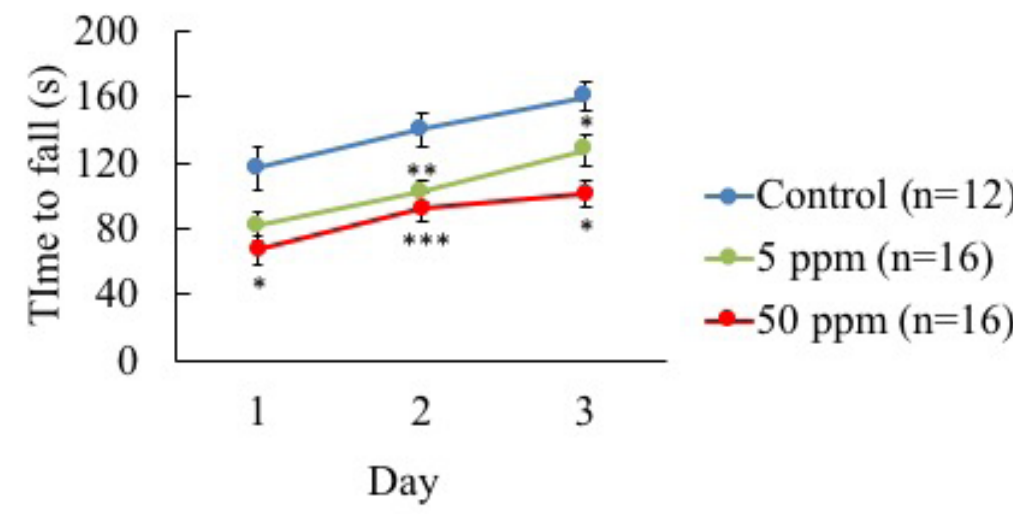

Figure 3. Rotarod Test

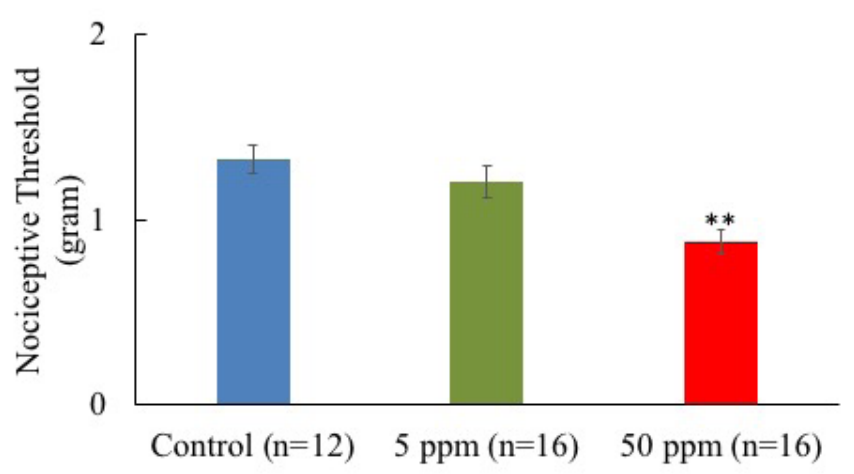

Figure 4. von Frey Hair Test

Regarding to the result, further study is fully required to link motor coordination, abnormal cerebellar development, and hypersensitivity.

\section{Conclusion}

Perinatal PTU-induced hypothyroid impaired motor coordination in adult female offspring. Additionally, we also found that hypothyroidism induced hypersensitivity that may contribute in motor coordination impairment. However, to clarify these issues, additional study is required.

\section{Acknowledgement}

We would like to thank the members of Department of Integrative Physiology, Gunma University Graduate School of Medicine for technical assistance and advance.

\section{Funding}

This study was supported in part by Grant-inAid for Scientific Research (No. 25281024) to NK from the Japanese Ministry of Education, Culture, Sports, Science and Technology (MEXT).

\section{Conflict of Interest}

None declared.

\section{References}

1. Amano I, Takatsuru Y, Khairinisa MA, Kokubo M, Haijima A, Koibichi N. Effects of mild perinatal hypothyroidism on cognitive function of adult male offspring. Endocrinology. 2018;159(4):1910-1921

2. Khairinisa MA, Takatsuru Y, Amano I, Kokubo M, Haijima A, Miyazaki W, Koibuchi N. In utero 
and postnatal propylthiouracilinduced mild hypothyroidism impairs maternal behavior in mice. Frontiers of Endocrinology. (Lausanne). 2018;9(5):1-11

3. Zimmermann MB: Iodine deficiency. Endocrinology Reviews. 2009;30:376408.

4. Zoeller RT, Rovet J: Timing of Thyroid Hormone Action in the Developing Brain: Clinical Observations and Experimental Findings. Journal of Neuroendocrinology. 2004;16:809-818

5. Morreale De Escobar G, Obregon MJ, Escobar Del Rey F. Role of thyroid hormone during early brain development. European Journal of Endocrinology. 2004;151 Suppl:U25-37

6. Koibuchi N, Jingu H, Iwasaki T, Chin WW. Current perspectives on the role of thyroid hormone in growth and development of cerebellum. Cerebellum. 2003;2:279-289

7. Hasebe M, Matsumoto I, Imagawa $T$, Uehara M. Effects of an anti-thyroid drug, methimazole, administration to rat dams on the cerebellar cortex development in their pups. International Journal of Development Neuroscience. 2008;26:409-414.

8. Shimokawa N, Yousefi B, Morioka S, Yamaguchi S, Ohsawa A, Hayashi H, Azuma A, Mizuno H, Kasagi M, Masuda H, Jingu H, Furudate SI, Haijima A, Takatsuru Y, Iwasaki t, Umezu M, Koibuchi N. Altered cerebellum development and dopamine distribution in a rat genetic model with congenital hypothyroidism. Journal of. Neuroendocrinology. 2014;26(3):164175

9. Reinehr T. Obesity and thyroid function. Molecular Cell of Endocrinology. 2010;316(2):165-71.

10. Mullur R, Liu YY, Brent GA. Thyroid hormone regulation of metabolism. Physiology Review. 2014;94(2):355-82.

11. Hoogwerf BJ, Nuttall FQ. Long-term weight regulation in treated hyperthyroid and hypothyroid subjects. American Journal of Medicine. 1984; 76(6):96370.

12. Gilbert ME. Impact of low-level thyroid hormone disruption induced by propylthiouracil on brain development and function. Toxicological Science. 2011;124(2):432-445

13. Koibuchi N. The role of thyroid hormone on functional organization in the cerebellum. Cerebellum. 2013;12(3):304-306

14. Alves IG, da Cruz KM, Mota CM, de Santana DS, Gaujac DP, de Carvalho VC, Reis LC, Sluka KA, QuintansJunior LJ, Antoniolli AR, Desantana JM, Badauê-Passos D Jr, de SantanaFilho VJ: Experimental hypothyroidism during pregnancy affects nociception and locomotor performance of offspring in rats. European Journal of Pain. 2013, 17:1291-1298

15. Refsgaard, LK, Hoffmann-Petersen J, Sahlhol M, Pickering DS, Andreasen JT. Modelling affective pain in mice: effects of inflammatory hypersensitivity on place escape/avoidance behaviour, anxiety and hedonic state. Journal of Neuroscience Methods. 2016;262, 85-92. 